\title{
Limits on coupling between dark components
}

\author{
Roberto Mainini, Silvio Bonometto \\ Department of Physics G. Occhialini - Milano-Bicocca University, Piazza della \\ Scienza 3, 20126 Milano, Italy \& I.N.F.N., Sezione di Milano
}

\begin{abstract}
DM-DE coupling can be a phenomenological indication of a common origin of the dark cosmic components. In this work we outline a new constraint to coupled-DE models: the coupling can partially or totally suppress the Meszaros effect, yielding transfered spectra with quite a soft bending above $k_{h o r, e q}$. Models affected by this anomaly do not show major variation in the CMB anisotropy spectrum and it is herefore hard to reconcile them with both $\mathrm{CMB}$ and deep sample data, through the same value of the primeval spectral index.
\end{abstract}

PACS numbers: 98.80.-k, 98.65.-r 


\section{Introduction}

There can be little doubts that a tenable cosmological model must include at least two dark components, cold Dark Matter (DM) and Dark Energy (DE); yet only hypotheses on their nature exist, most of them assuming that DM and DE are physically unrelated and that their similar densities, in today's world and just in it, are purely accidental.

Attempts to overcome this conceptual deadlock were made by several authors suggesting, first of all, that DE has a dynamical nature [1] (for a review see [2] and references therein). An alternative idea is that DE is a phenomenological consequence of the emergence of nonlinearity; this appealing option was repeatedly considered (see, e.g., [3] and references therein), but is far from being shown and leaves however apart the question of DM nature. Interactions between DM and dynamical DE [4] (see also [5]) might partially cure the problem, keeping close values for their densities up to large redshift. This option could also be read as an approach to a deeper reality, whose physical features could emerge from phenomenological limits to coupling strength and shape.

A longer step forward was attempted by [6], suggesting that DM and DE derive from a single complex scalar field, being its quantized phase and modulus, respectively. The complex field could be the one responsible for $\mathcal{C P}$ conservation in strong interactions, within a scheme similar to Peccei \& Quinn framework [7] (see also [8]). At variance from previous suggestions, which introduce parameters and aim at limiting them through data fitting, this option - dubbed dual-axion model-cuts the available degrees of freedom, including as many parameters as a standard-CDM approach. It is then quite appealing that its reduced parameter budget is sufficient to fit quite a number of observational constraints [9], still allowing for a common nature of DM and DE and for a specific shape of interaction between them.

In this paper, however, we keep on the phenomenological side and discuss generic constraints to DM-DE interactions. This discussion will have a fallout also on the dual-axion approach, which does face a problem, because of the feature of the DM-DE coupling it causes.

A coupling of baryons with DE is ruled out by observational consequences similar to modifying gravity. Limits are looser for DM-DE coupling, whose consequences can be appreciated only over cosmological distances. It should also be outlined that forces acting within the dark sector could modify predictions on high concentration DM lumps. There can be little doubts that cold DM particles, feeling gravity only, give them NFW profiles. Yet, observational data do not lend much support to this shape, for any scale range, and direct interaction between DM particles is severely constrained also by recent data.

This is a further reason to consider DM-DE interactions, either as a fundamental theory or as an effective framework to approach deeper physics. It is then important to devise any observational limit to such interactions and, in this paper, we outline further constraints to its shape; they are consequences of the early behavior of density 
fluctuations, over scales destined to evolve into non-linear structures.

Fluctuations over such scales enter the horizon before matter-radiation equality and their growth is initially inhibited by the overwhelming density of the radiative component, then still behaving as a single fluid together with baryons. While fluctuations in the fluid behave as sonic waves, self gravitation of DM is just a minor dynamical effect is respect to cosmic expansion. This freezing of fluctuation amplitudes until equality is known as Meszaros effect.

The main point we wish to outline here is that DM-DE coupling can damp Meszaros effect, so that the rate of fluctuation growth, between the entry in the horizon and equality, is significantly enhanced. As a matter of fact, fluctuation freezing is essential, in shaping the transfered spectrum, which peaks on the scale $k_{h o r, e q}$ entering the horizon at equality. At smaller mass scales $\left(k>k_{h o r, e q}\right)$ the spectrum declines because of the increasing duration of the freeze.

The freezing or its damping have modest consequences on the evolution of fluctuations in baryons and radiation, evolving then in the sonic regime. What we shall therefore find are significant changes in the transfer funcions, while CMB spectra keep almost unaffected.

Constraints to coupled DE models arise from both linear and non-linear effects. It has been known since long that coupling may cause a $\phi-M D$ epoch after matterradiation equality (see, e.g., [10]). This changes the (comoving) distance of the last scattering band. In order to fit data, the present value of the Hubble parameter $H_{o}$ needs then to be increased. Limits on $H_{o}$ turn then into limits to the coupling.

Limits to the coupling, in the case of a Ratra Peebles [11] self-interaction potential, where also found in [12], by studying halo concentration distibution.

The feature outlined in this work affects the transfer function, leaving almost unaffected CMB anisotropies. Discussing how the transfer function is affected by DMDE coupling is the main aim of this technical paper. We shall also exhibit CMB angular spectra, to confirm that they suffer just marginal changes.

No general data fitting, constraining parameters and/or showing specific model advantages, will be made here. In fact, what we wish to outline is a major effect, which allows to discard a class of models, a priori. This is why we keep to cosmological parameter values ensuing from WMAP3 best-fit [13], although deduced by assuming a $\Lambda$ CDM model. In particular, we shall take an overall density parameter $\Omega=1$; the present value of the cold DM (baryon) density parameter will be $\Omega_{o, c}=0.224$ $\left(\Omega_{o, b}=0.044\right)$; the dimensionless Hubble parameter will be $h=0.704$; the primeval spectral index, when not taken as a free parameter, will be $n=0.947$.

Within this frame we shall consider a self-interacting scalar field, causing cosmic acceleration when its pressure/density ratio $w=p_{D E} / \rho_{D E}$ falls in the range $(-1,-1 / 3)$. Quite in general, it is

$$
\rho_{D E}=\rho_{k, D E}+\rho_{p, D E} \equiv \dot{\phi}^{2} / 2 a^{2}+V(\phi), \quad p_{D E}=\rho_{k, D E}-\rho_{p, D E},
$$


So that it is $-1 / 3 \gg w>-1$ when dynamical equations yield $\rho_{k, D E} / V \ll 1 / 2$. Here

$$
d s^{2}=g_{\mu \nu} d x^{\mu} d x^{\nu}=a^{2}(\tau)\left(-d \tau^{2}+d x_{i} d x^{i}\right) \quad(i=1, . ., 3)
$$

is the background metric and dots indicate differentiation with respect to $\tau$ (conformal time). The $w$ ratio exhibits a time dependence set by the shape of $V(\phi)$. Much work has been done on dynamical DE (see, e.g., [2] and references therein), also aiming at restricting the range of acceptable $w(\tau)$ 's, so gaining an observational insight onto the physics responsible for the potential $V(\phi)$.

Our analysis here will however be restricted to SUGRA potentials [14]

$$
V(\phi)=\left(\Lambda^{\alpha+4} / \phi^{\alpha}\right) \exp \left(4 \pi \phi^{2} / m_{p}^{2}\right)
$$

admitting tracker solutions. Here $m_{p}=G^{-1 / 2}$ is the Planck mass. This will enable us to focus on peculiarities caused by the coupling. Let us also remind that, once the DE density parameters $\Omega_{D E}$ is assigned, either $\alpha$ or the energy scale $\Lambda$, in the potentials (3), can still be freely chosen. In this paper we show results for $\Lambda=10^{2} \mathrm{GeV}$.

The SUGRA potential, at least in the absence of coupling, yields an excellent fit of observational data [15]. We tested the effects of coupling for a number of values of the scale $\Lambda$, from 10 to $10^{4}$, and also changing the shape of the potential into Ratra-Peebles.

In the former case we find just marginal shifts. In the latter one, quantitative changes can be significant. The overall behavior is however identical and this potential is known to yield a poor fit to CMB data, unless quite a small $\Lambda$ scale is taken, so spoiling its physical appeal.

Within this frame we aim at focusing problems and showing the quantitative consequences of different options.

\section{Dynamical equations}

Let us then start from the background equations for DE and DM when the metric is (2), reading

$$
\ddot{\phi}+2 \frac{\dot{a}}{a} \dot{\phi}+a^{2} V_{, \phi}=C(\phi) a^{2} \rho_{c}, \quad \dot{\rho}_{c}+3 \frac{\dot{a}}{a} \rho_{c}=-C(\phi) \dot{\phi} \rho_{c}
$$

where we set

$$
C(\phi)=4 \sqrt{\frac{\pi}{3}} \frac{\beta}{m_{p}}\left(\frac{\phi}{m_{p}}\right)^{\epsilon}=4 \sqrt{\frac{\pi}{3}} \frac{\tilde{\beta}}{m_{p}}
$$

A possible time dependence of the coupling strength was considered but not deepened since the early work of [19]. The dual-axion model naturally predicts a coupling $C=1 / \phi$, consistent with eq. (5), if $\epsilon=-1$ and $\beta \simeq 0.244$. Quite in general, for dimensional reasons, $C$ can be expressed through products of $m_{p}^{a}$ and $\phi^{b}$ with $a+b=-1$. During most cosmic evolution $\phi$ is a monotonically increasing function, so that the sign of the exponent of $\phi$ tells us whether the coupling was stronger of weaker in the past. (An exception can be recent times, as the exponential term in the SUGRA potential can cause a re-bounce of $\phi$ when it approaches $m_{p}$; our arguments here concern much earlier times, when it is safely $\phi<m_{p}$ ). 
An expression of $C$, made by a polynomial including terms with different powers of $\phi$, could select a peculiar epoch to have then a weaker or stronger coupling. Such an option, however, is clearly $a d-h o c$ and does not seem to deserve further investigation.

An explicit dependence of $C$ upon time would be hard to reconcile with the Lorentz invariance of the Lagrangian mass term

$$
\mathcal{L}_{c}=-B(\phi) m_{\chi} \bar{\chi} \chi
$$

setting the coupling between the DE scalar field $\phi$ and a spinor field $\chi$ supposed to yield DM, as $C(\phi)=d(\ln B) / d \phi$. (Notice that $B(\phi) m_{\chi}$ is the time-dependent mass of DM quanta). A dependence of $C$ on $\phi$ seems therefore the only way to instaure a time-dependent coupling.

Let us then describe fluctuation equations. In the period when Meszaros effect occurs, DM, photons and baryons can be treated as fluids; (massless) neutrinos, instead, are not a fluid. Fluctuations in a generic fluid with $p / \rho=w$ and $\delta p / \delta \rho=c_{s}^{2}$ fulfill the equations

$$
\begin{aligned}
& \dot{\delta}=-(1+w)(k v+\dot{h} / 2)-3\left(c_{s}^{2}-w\right)(\mathcal{H}-C \dot{\phi}) \delta-(1-3 w)\left(C \dot{\varphi}+C^{\prime} \dot{\phi} \varphi\right) \\
& \dot{v}=-(1-3 w)(\mathcal{H}-C \dot{\phi}) v+\frac{c_{s}^{2}}{1+w} k \delta-\frac{\dot{w}}{1+w} v-k \sigma-k C \frac{1-3 w}{1+w} \varphi .
\end{aligned}
$$

Here $\delta=\delta \rho / \rho, v=i u_{i} k^{i} / k,(\rho+p) \sigma=-\left(\hat{k}_{i} \cdot \hat{k}_{j}-\delta_{i j}\right)\left(T^{i j}-\delta_{i j} T_{k}^{k} / 3\right)\left(u_{i}\right.$ are the space components of the velocity field in the fluid and $T^{i j}$ is its stress-energy tensor) and $\mathcal{H}=\dot{a} / a$, while the DE field

$$
\phi(\tau, \mathbf{x})=\phi_{o}(\tau)+\varphi(\tau, \mathbf{x})
$$

is split into a background component $\phi_{o}$, coinciding with the $\phi$ field obeying the eq. (4), and the space-dependent fluctuation $\varphi$; in eqs. (7), Fourier components of $\varphi$ are considered which fulfill the equation:

$$
\ddot{\varphi}+2 \mathcal{H} \dot{\varphi}+k^{2} \varphi+a^{2} V_{\phi}^{\prime \prime} \varphi+\dot{\phi} \dot{h} / 2=C a^{2} \rho_{c} \delta_{c}+C_{\phi}^{\prime} a^{2} \rho_{c} \varphi,
$$

while fluctuation self-gravity is fully accounted by $\dot{h}$, obtained by integrating the equation

$$
\ddot{h}+\mathcal{H} \dot{h}=-8 \pi G\left\{a^{2} \rho\left[\Omega_{\gamma b}\left(1+3 c_{s}^{2}\right) \delta_{\gamma b}+\Omega_{c} \delta_{c}\right]-2 a^{2} V_{\phi}^{\prime} \varphi+\dot{\phi} \dot{\varphi}\right\}
$$

In the absence of coupling $(C=0)$, we can take $v=0$ in eqs. (7) without loss of precision, and face the dynamical problem though a single first order equation. This is no longer true when DM particles can be pushed by DE forces. Then the DM equation, on scales below the horizon and on times before matter-radiation equality, reads

$\ddot{\delta}_{c}+\left[\frac{\dot{a}}{a}-\frac{4}{m_{p}} \sqrt{\frac{\pi}{3}} \tilde{\beta}(\phi) \dot{\phi}\right] \dot{\delta}_{c}-4 \pi G a^{2} \rho\left\{\left[1+\frac{4}{3} \tilde{\beta}^{2}(\phi)\right] \Omega_{c} \delta_{c}+\Omega_{\gamma b} \delta_{\gamma b}\left[1+3 c_{s}^{2}\right]\right\}=0$,

Here, $\rho$ is the overall density; $\Omega_{c}$ and $\Omega_{\gamma b}$ are time dependent density parameters for DM and the baryon-photon fluid. To show eq. (11) one needs a little algebra, which will be postponed to the end of the section. 
Still from eqs. (7), we can also work out the equations for the photon-baryon fluid, by taking $C=0$ and

$$
\begin{gathered}
c_{s}^{2} \equiv \delta p_{\gamma} /\left(\delta \rho_{b}+\delta \rho_{\gamma}\right)=\left[3\left(1+3 \Omega_{b} / 4 \Omega_{\gamma}\right)\right]^{-1} \\
w_{\gamma b}(a) \equiv p_{\gamma} /\left(\rho_{b}+\rho_{\gamma}\right)=\left[3\left(1+\Omega_{b} / \Omega_{\gamma}\right)\right]^{-1} .
\end{gathered}
$$

Here $\Omega_{b}$ and $\Omega_{\gamma}$ are the time dependent baryon and photon density parameters. The baryon component can be responsible for a shift of $w_{\gamma b}$ from $1 / 3$; although initially small, it can approach 20-25\% at the eve of recombination. Notice then that a non-vanishing factor $1-3 w \sim \Omega_{b} / \Omega_{\gamma}$ keeps a direct influence of $\varphi$ on baryon-photon fluctuations.

This set of equations enables the reader to build a simplified numerical algorithm, directly testing the suppression of Meszaros' effect.

Clearly, to study the later evolution, since the eve of baryon-photon decoupling a full kinetic treatment of the radiation is needed. This will be used to confirm that CMB anisotropies are just marginally affected, but is unessential to focus on the greater changes occurring to the transfer function.

Let us now summarize the procedure to obtain eq. (11); a reader unintersted in it can skip the rest of this section.

The starting point are again the eqs. (7). Together with them, let us consider again eq. (9). There, any mass-like term multiplying $\phi$, in comparison with $k^{2}$, is negligible; then, before equality, it yields

$$
\ddot{\varphi}+(2 / \tau) \dot{\varphi}+k^{2} \varphi=C a^{2} \rho_{c} \delta_{c}-\dot{\phi} \dot{h} / 2
$$

Here, two kinds of time dependence must be compared, over fluctuation and Hubble time scales. Accordingly, we can express $\varphi$ as sum of rapidly and slowly varying terms by actually summing up the (rapidly varying) general integral of the equation obtainable by equating to zero the l.h.s. and a (slowly varying) integral obtained by equating the last term at the 1.h.s. with the r.h.s. . If we then time-average over fluctuation time scales, only the latter contribution survives and

$$
\langle\varphi\rangle \simeq \frac{1}{k^{2}}\left(C a^{2} \rho_{c} \delta_{c}-\dot{\phi} \dot{h} / 2\right)
$$

Let us recall that dropping the contribution of fluctuating terms is the standard procedure to obtain an analytical description of Meszaros' effect. The point here is that, while all $\varphi$ derivatives can be dropped, there is a slowly varying contribution to $\varphi$ which cannot be soon disregarded.

However, if the expression (15) is used to replace $\varphi$ in eqs. (7) for CDM, yielding

$$
\dot{\delta}_{c}+k v_{c}+\frac{\dot{h}}{2}=-2 \beta^{2} \frac{d}{d \tau}\left[\left(\frac{\phi}{m_{p}}\right)^{2 \epsilon} \frac{\Omega_{c} \delta_{c}}{(k \tau)^{2}}\right]+4 \sqrt{\frac{\pi}{3}} \frac{\beta}{k^{2}} \frac{d}{d \tau}\left(\frac{\dot{\phi} \dot{h}}{\phi^{-\epsilon} m_{p}^{1+\epsilon}}\right)
$$

it becomes clear that also the slowly varying $\varphi$ contributions can be dropped. In fact, the first term at the r.h.s. contains a division by $(k \tau)^{2}$, which is the squared ratio between long and short timescales and, altogether, it is $\sim \mathcal{O}\left[\dot{\delta} /(k \tau)^{2}\right] \ll \dot{\delta}$. We must then acknowledge that a time derivative of a quantity $Q$, varying over the Hubble time 
scale, is $\sim \mathcal{O}(Q / \tau)$. The second term at the r.h.s. is then $\sim \mathcal{O}\left[\left(\phi / m_{p}\right)^{1+\epsilon} \dot{h} /(k \tau)^{2}\right]$, while we took $1+\epsilon>0$ and, in the epoch considered, $\phi / m_{p} \ll 1$. It must then be even smaller than the the first one.

Setting then to zero the 1.h.s. of eq. (16), we obtain the relations

$$
\ddot{\delta}_{c}+k \dot{v}_{c}+\frac{\ddot{h}}{2} \simeq 0, \quad k v_{c} \simeq-\dot{\delta}_{c}-\frac{\dot{h}}{2} .
$$

In turn, the second eq. (7), using this latter equality, yields the relation

$$
k \dot{v}_{v}=(C \dot{\phi}-\mathcal{H})\left[-\dot{\delta}_{c}-\dot{h} / 2\right]-k^{2} C \varphi
$$

which can be replaced in the former eq. (17), together with eq. (10) and (15), obtaining

$$
\ddot{\delta}_{c}+[\mathcal{H}-C \dot{\phi}] \dot{\delta}_{c}-4 \pi G\left\{a^{2} \rho\left[\Omega_{\gamma b}\left(1+3 c_{s}^{2}\right) \delta_{\gamma b}+\Omega_{c} \delta_{c}\right]-2 a^{2} V_{\phi}^{\prime} \varphi+\dot{\phi} \dot{\varphi}\right\}-C^{2} a^{2} \rho_{c} \delta_{c}=0
$$

Neglecting here $\varphi$ fluctuations as source terms - DE yields a minor contribution to the overall density, as shown in Fig. 6- and using eq. (5), eq. (11) is soon obtainable.

\section{Overcoming the freeze}

Let us then focus our attention on eq. (11) and outline first that, in average, the term $\Omega_{\gamma b} \delta_{\gamma b}\left(1+3 c_{s}^{2}\right)$ almost vanishes, as sonic waves fluctuate. Then, in the absence of coupling $(\tilde{\beta}=0)$, the self-gravitation term $\propto \delta_{c}$ is also damped by $\Omega_{c} \ll 1$. If the photon-baryon term is then neglected, the increasing mode (approximately) reads

$$
\delta_{c} \propto 1+3 y / 2
$$

with $y=3 w_{\gamma b} a / a_{e q}$ (see, e.g., [16]) and this yields a growth from horizon to equality never exceeding a factor 2.5, that we shall approximate as $\delta_{c} \propto a^{1 / 4}$, according to numerical outputs. Meanwhile, above the horizon, in a synchronous gauge, $\delta_{c} \propto a^{2}$. Hence, for $k>k_{h o r, e q}$, the growth is slowed down by a factor $\propto a_{h}^{7 / 4}(k)$, while the scale factor when $k$ passes through the horizon, $a_{h}(k) \propto k^{-1}$. Altogether, for $k>k_{\text {hor }, e q}$, we expect a transfered spectrum $P(k)=A k^{n} \mathcal{T}^{2}(k) \simeq A k^{n-3.5}(\mathcal{T}(k)$ is the transfer function).

It is then easy to see what can change because of the coupling. The coefficient of the friction term $\propto \dot{\delta}_{c}$ is certainly reduced and can even invert its sign. Even more significantly, the term $\left[1+4 \tilde{\beta}^{2}(\phi) / 3\right] \Omega_{c}$ can attain or overcome unity, not only because of the greater size of $\tilde{\beta}(\phi)$, but also thanks to the modified time dependence of $\Omega_{c}$. It turns out, in fact, that a decreasing dependence of $\tilde{\beta}$ on $\phi(\epsilon<0)$ yields higher $\Omega_{c}$ values in the relevant redshift interval. In general, $\phi$ is smaller at earlier times and $\epsilon<0$ causes a stronger coupling in the past.

Let us however debate first the constant coupling case $(\epsilon=0)$. Available data set then a constraint $\beta<0.1-0.2$ [10], [12] (see also [17]; beware of the different coupling definition), limiting the acceptable discrepancy of DM and DE evolution, after recombination, from uncoupled models. The most direct effect, in this case, concerns large scales entering the horizon late, when such discrepancies occur. The low- $l$ plateau 


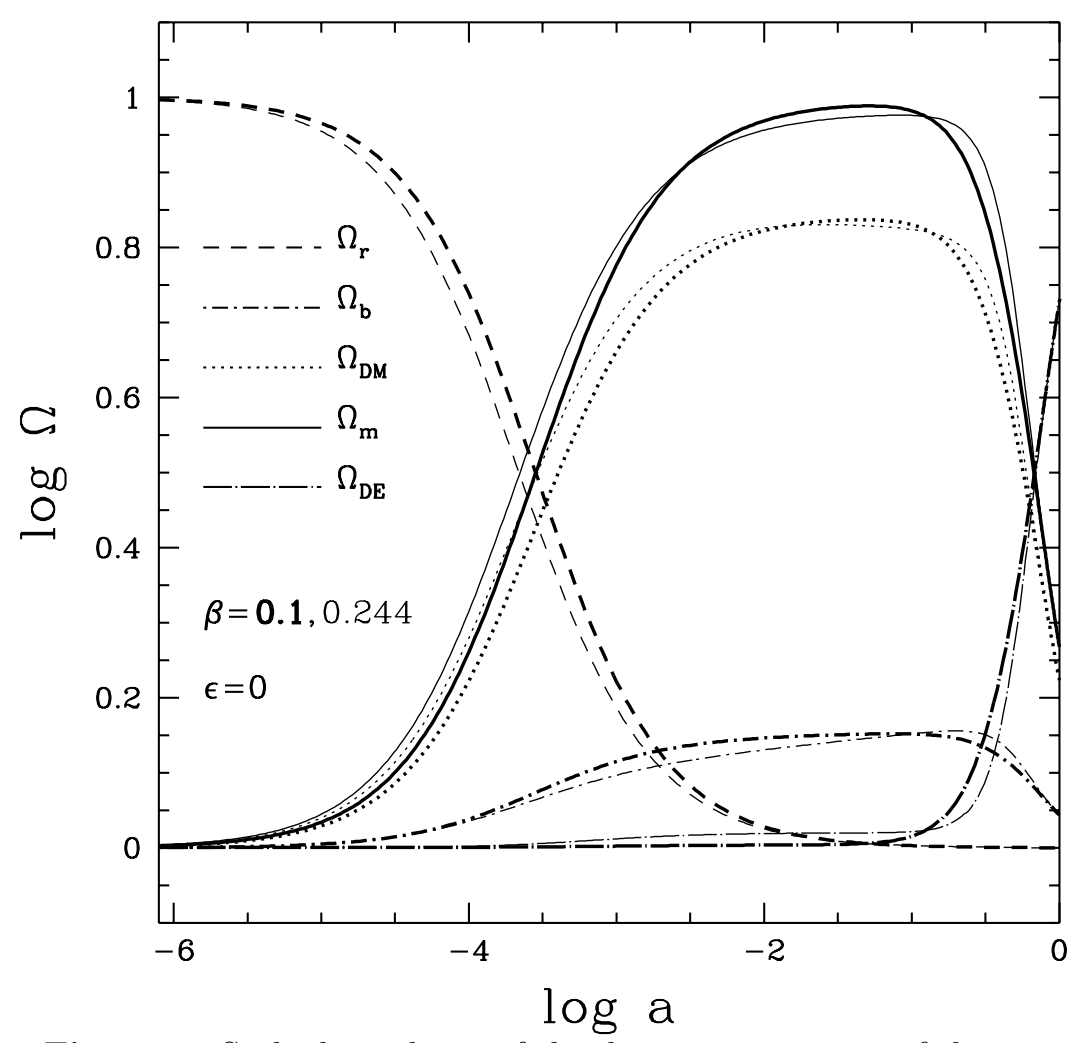

Figure 1. Scale dependence of the density parameters of the various components in coupled DE models with constant coupling. This plot shows also the displacement of $z_{e q}$ and, henceforth, of $k_{h o r, e q}$ as $\beta$ increases: thicker (thinner) curves refer to $\beta=0.01$ (0.0244).

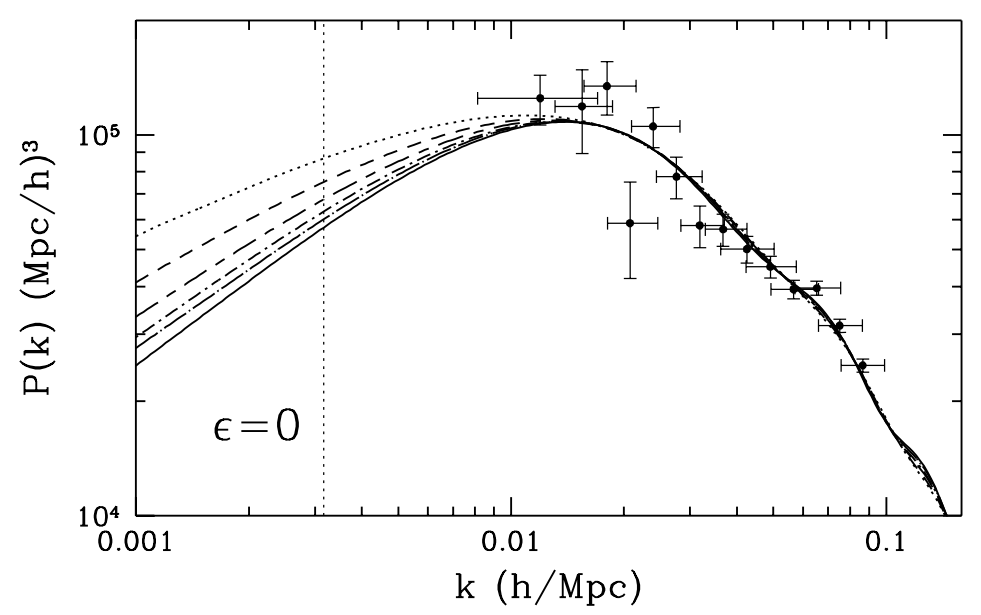

Figure 2. Best fits of SDSS data for constant $\beta$ from 0 (solid line) to 0.25 (dotted line). Different lines correspond to a $\beta$ increase by 0.05 . The vertical dotted line yields the scale of $C_{10}$. 


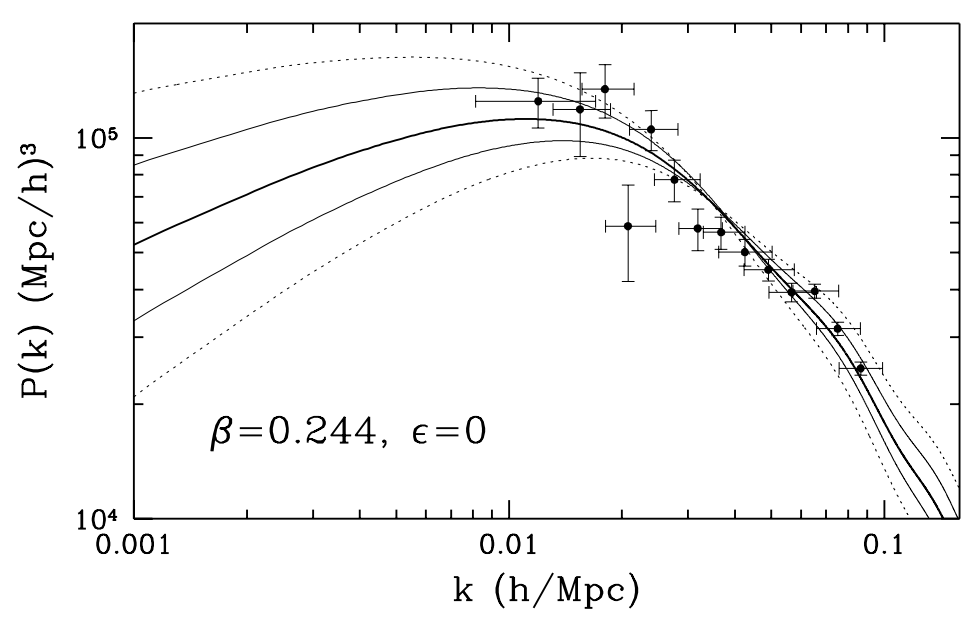

Figure 3. If values of $n$ at $1-$ or $2-\sigma$ 's from best fits are taken, spectra are significantly modified. Here we show the effect in the case with $C=1 / m_{p}$.

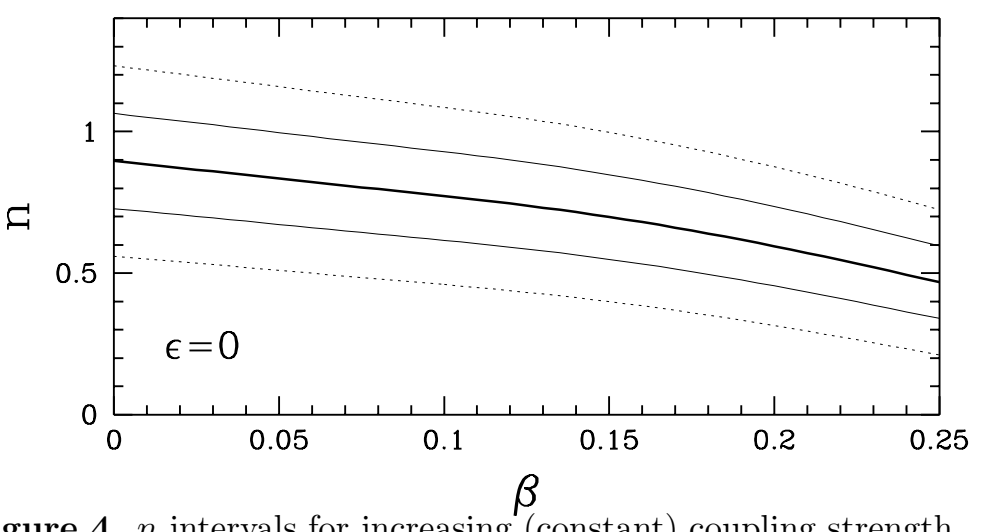

Figure 4. $n$ intervals for increasing (constant) coupling strength

of the CMB anisotropy spectrum can then undergo a modified ISW effect, while some changes in the $C_{l}$ behavior, up to the first peak at $l \sim 200$, can be compensated by slightly modifying $n, h$ and other parameters.

But the most significant shifts occur on the transfer function. Its slope, at $k>k_{h o r, e q}$ and up to a scale $k \sim 0.1 / \mathrm{hMpc}^{-1}$, where non-linearity effects become important, is slightly distorted as $\beta$ increases. The main effect, however, is a progressive displacement of $k_{\text {hor, eq }}$ itself. In Figure 1 we compare the $\Omega$ evolution in models with different constant coupling, showing the significant displacement of the crossing between $\Omega_{r}$ and $\Omega_{c}$, when different $\beta$ 's are taken.

We can show the impact of this displacement by fitting transfered spectra, over these scales, with the Luminous Red Galaxies sample data from the Sloan Digital Sky Survey (SDSS) [18] and allowing the primeval spectral index $n$, assumed to be constant, to act as a free parameter. 
In Figure 2 we show the result of this fit. Transfered spectra, when $k_{\text {hor }, e q}$ vary, easily accommodate deep sample data in the linear range, as the $k_{h o r, e q}$ shift is compensated by a slightly smoother slope. All that however requires a non negligible decrease of $n$, and transfered spectra risk to become too high in the region where they should fit CMB data (the scale of the 10-pole is indicated in the Figure).

An attempt to balance this spectral distortion can be made by varying other parameters. It is then significant to consider Figure 3, showing deep sample data vs. transfered spectra computed with $n$ 's within $1-$ and $2-\sigma$ 's from the best-fit. In the Figure we took $\beta=\sqrt{3 / \pi} / 4 \simeq 0.244$. Then, in Figure 4, we show the $1-$ and $2-\sigma$ range of $n$, for constant coupling strength. Let us also recall that likelihood analysis showed that models with constant $\beta<\sim 0.2$ do not exhibit severe disagreements with data.

The effect on CMB data fitting arising from $n$ values distant from unity can be also directly inspected in Figure 5. According to it, we can examine Fig. 4 assuming to be viable only those models which, at the $1-\sigma$ level, admit $n>\sim 0.85$. With reference to this admittedly qualitative criterion, we shall now consider the variable coupling case.

First of all, when coupling varies, the evolution of the density parameters exhibits significant discrepancies from the constant coupling behavior. The point is that, at high redshift, they further strengthen DM self-gravity, coherently with higher $\tilde{\beta}$ effects. Results of a numerical integrations, illustrating this issue, are shown in Figure 6, where we compare the redshift dependence of density parameters in constant $(\epsilon=0)$ and strongly variable $(\epsilon=-1)$ coupling models, allowing to appreciate a substantial enhancement of $\Omega_{c}$ at the eve of equality. Altogether $\left[1+4 \tilde{\beta}^{2}(\phi) / 3\right] \Omega_{c}$ is significantly greater, and the freeze of $\delta_{c}$, between horizon entry and equality, is almost canceled.

This explains the behaviors shown in Figure 7, which exhibit one of the main findings of this work. These plots are obtained from a numerical evaluations of $\delta_{c}$, in models with different $\beta$ for $\epsilon=0$ or -1 . In the former case, the high- $z$ effect of coupling is marginal. In the latter one, the fluctuation growth is substantially enhanced, more significantly for greater $k$ values.

Accordingly, we can expect modifications in the transfer function and in the fit of observational data. Our general conclusion is that a time dependence of DM-DE coupling, making it stronger at higher redshift, can prevent DM fluctuations to have a stationarity period after their entry in the particle horizon, so causing large modification of the transfer function.

\section{Results}

These expectations will be tested by using an algorithm solving the whole set of dynamical equations. To this aim we can use our extension of the public programs CAMB, or our own code, with identical outputs.

Results for transfer functions are shown in Fig. 8 for $\beta=0.1$ and 0.244 , and a variety of values of $\epsilon$. 


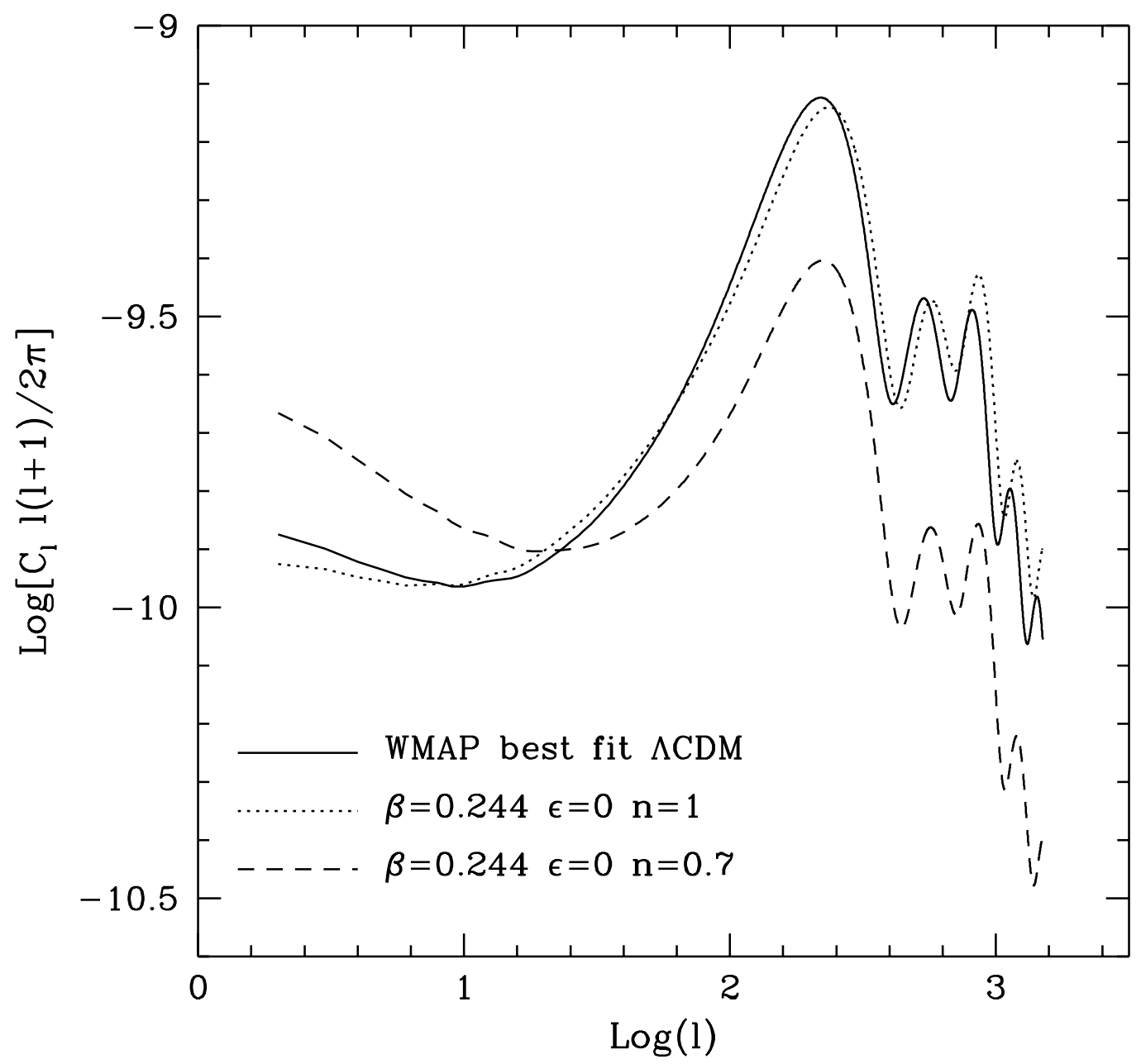

Figure 5. Anisotropy spectrum of the $\Lambda$ CDM model yielding the best fit to WMAP3 data compared with the spectra for coupled models with $\beta=0.1$ and $\epsilon=0$, for $n=1$ and $n=0.7$. Already in the former case some difference exists, but no major qualitative changes occur; by adjusting other model parameters one can expect a reasonable fit to data. Taking $n=0.7$, any fitting to CMB anisotropy data is apparently excluded.

The suppression of fluctuation freezing is obviously stronger for greater $\beta$ (and increasingly negative $\epsilon$ values). For $\epsilon=-1$, enclosing the case $C=1 / \phi$ when $\beta=0.244$, the steepness of the transfer function, for $k>k_{h o r, e q}$ is much reduced. The effect is still significant also for $\epsilon=-0.5$, namely when $\beta=0.244$.

A further effect shown by these plots is a significant displacement of the scale where $\mathcal{T}$ begins its gradual descent. As a consequence, different coupling laws may cause displacements on the scale where transfered spectra peak.

Notice that, while this occur, the CMB anisotropy spectum keeps quite a reasonable behavior, if $n \simeq 1$, as is shown in Figure 9, similar to Fig. 5 but for variable coupling; here we compare the WMAP3 data on the anisotropy spectrum with the spectra obtained 

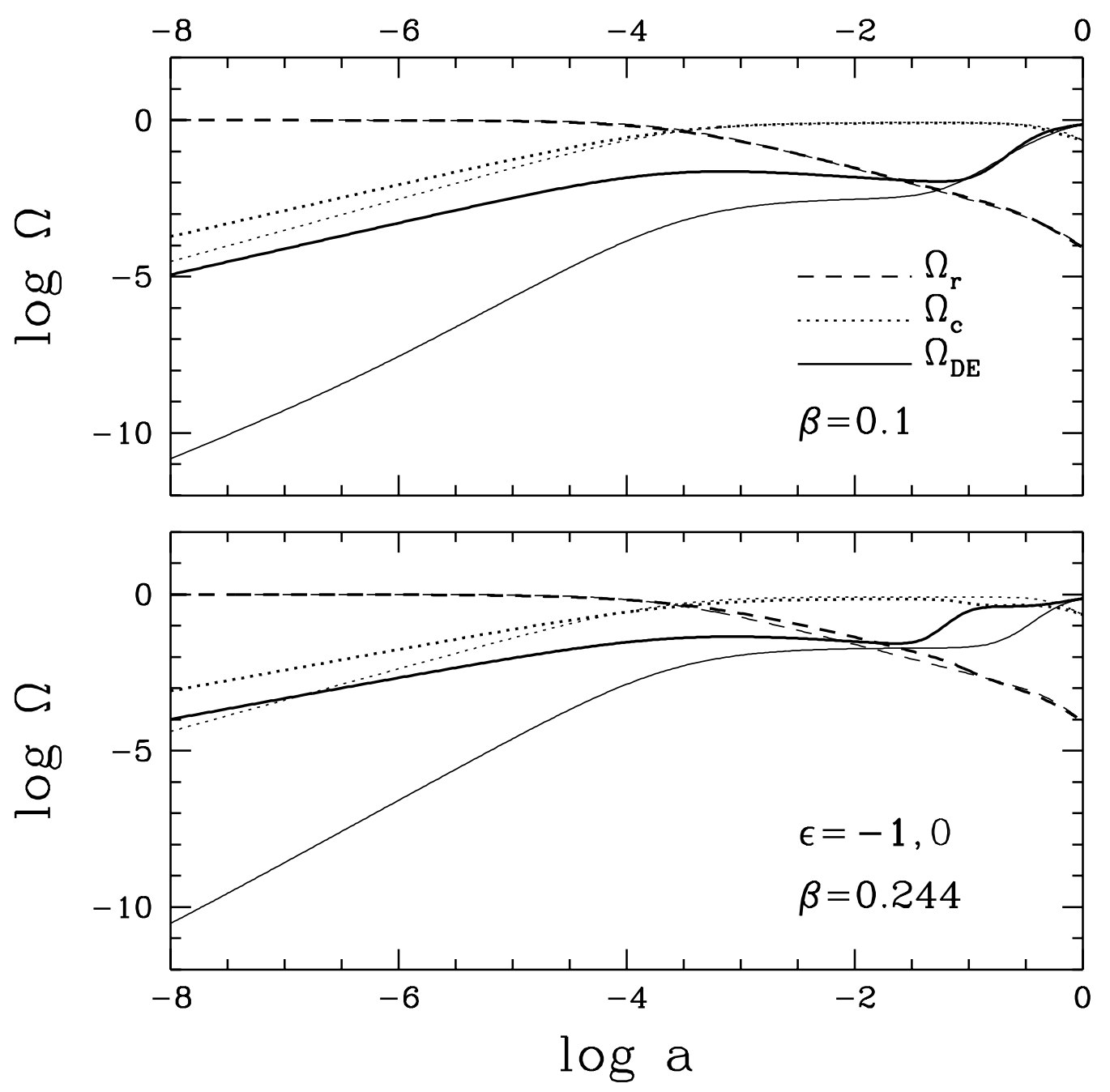

Figure 6. Redshift dependence of density parameters $\Omega$ in coupled DE models with constant and variable coupling. The two panels refer to different values of $\beta$. In both panels we show $\Omega$ 's for $\epsilon=0\left(C \propto \beta / m_{p}\right.$, thinner lines $)$ and $\epsilon=-1(C \propto \beta / \phi$, thicker lines). Figures are rather intricate and their reading may begin from dashed lines, yielding $\Omega_{r}=\Omega_{\gamma}+\Omega_{\nu}$, which are almost independent from the coupling law. A more relevant effect occurs on $\Omega_{c}$ (dotted lines), whose values, in the case of variable coupling, exceed those of constant coupling until equality. The most relevant effect occurs for DE (solid lines), whose contribution to the overall density is enhanced by several order of magnitude by variable coupling. DE fluctuations will fade after the entry in the horizon; however, high $\Omega_{D E}$ values increase their impact on other components before their disappearance. 

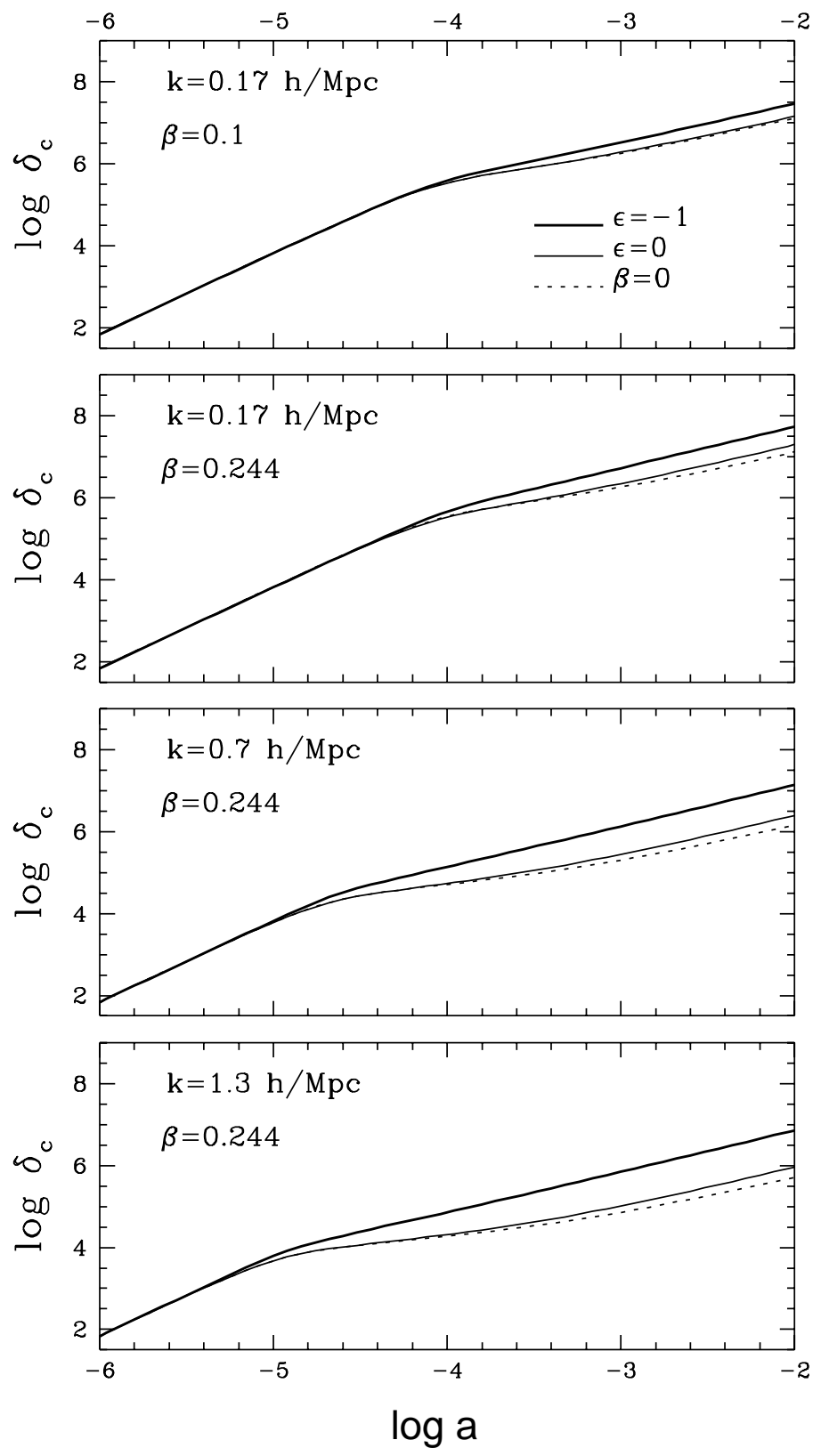

Figure 7. Evolution of DM fluctuations in a time interval enclosing the entry in the horizon and the matter-radiation equality. In all cases, in the presence of DM-DE coupling some modification occurs. They are however almost negligible for constant coupling, while, for coupling $\propto \phi^{-1}$, Meszaros' freezing is almost completely canceled. 


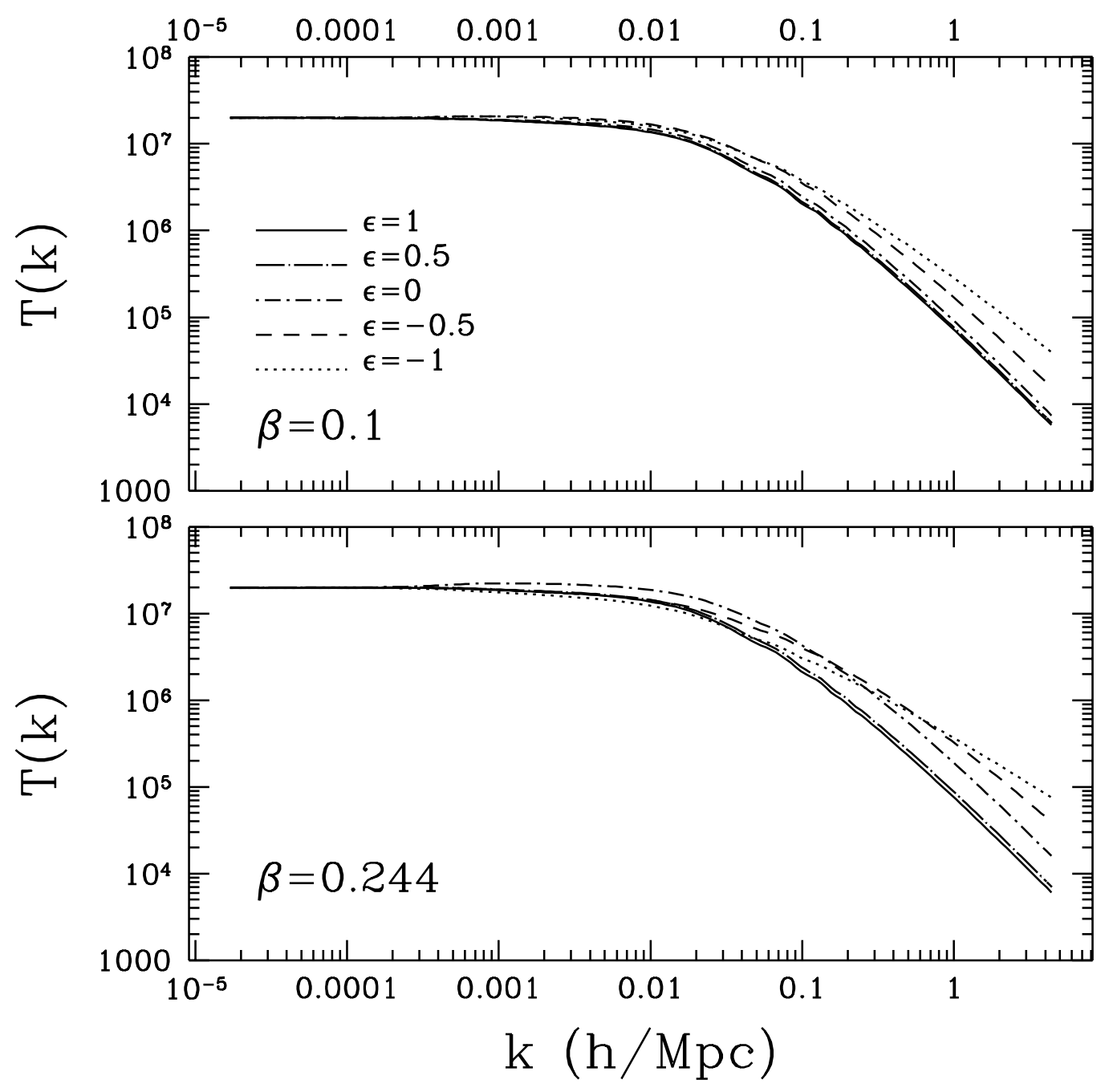

Figure 8. Transfer functions for different behaviors of DM-DE coupling with redshift and/or for different coupling normalization. The case $\epsilon=0$ corresponds to redshift independent coupling intensity. The case $\epsilon=-1$ with $\beta=0.244$ correspond to a coupling $C=1 / \phi$. Besides of the different slopes, notice the dependence on the model of the bending scale and, in particular, its dependence on the coupling strength, also in constant coupling models (dash-dotted lines).

for $\beta=0.1, \epsilon=-1$ and two $n$ values: $n=1$ apparently allowing a reasonable fit, and $n=0.7$, as needed to obtain a reasonable transfered spectrum. Figure 8 , in fact, is a direct evidence that, in order to recover a fair slope of the transfered spectrum in the scale range where structures accumulate, small primeval $n$ values are unavoidably required. In order to perform an evaluation of the effect, we actually built transfered spectra and compared them again with SDSS data. In this way we find $n \sim 0.5-0.7$. Fig. 9 shows that this spoils the fit with $C_{l}$ data.

Spectra with ordinary downward bending fit both deep sample and CMB data with $n \simeq 1$. On the contrary, spectra with low $n$ 's and standard $\sigma_{8}$ 's cause greater $C_{l}$ still in 


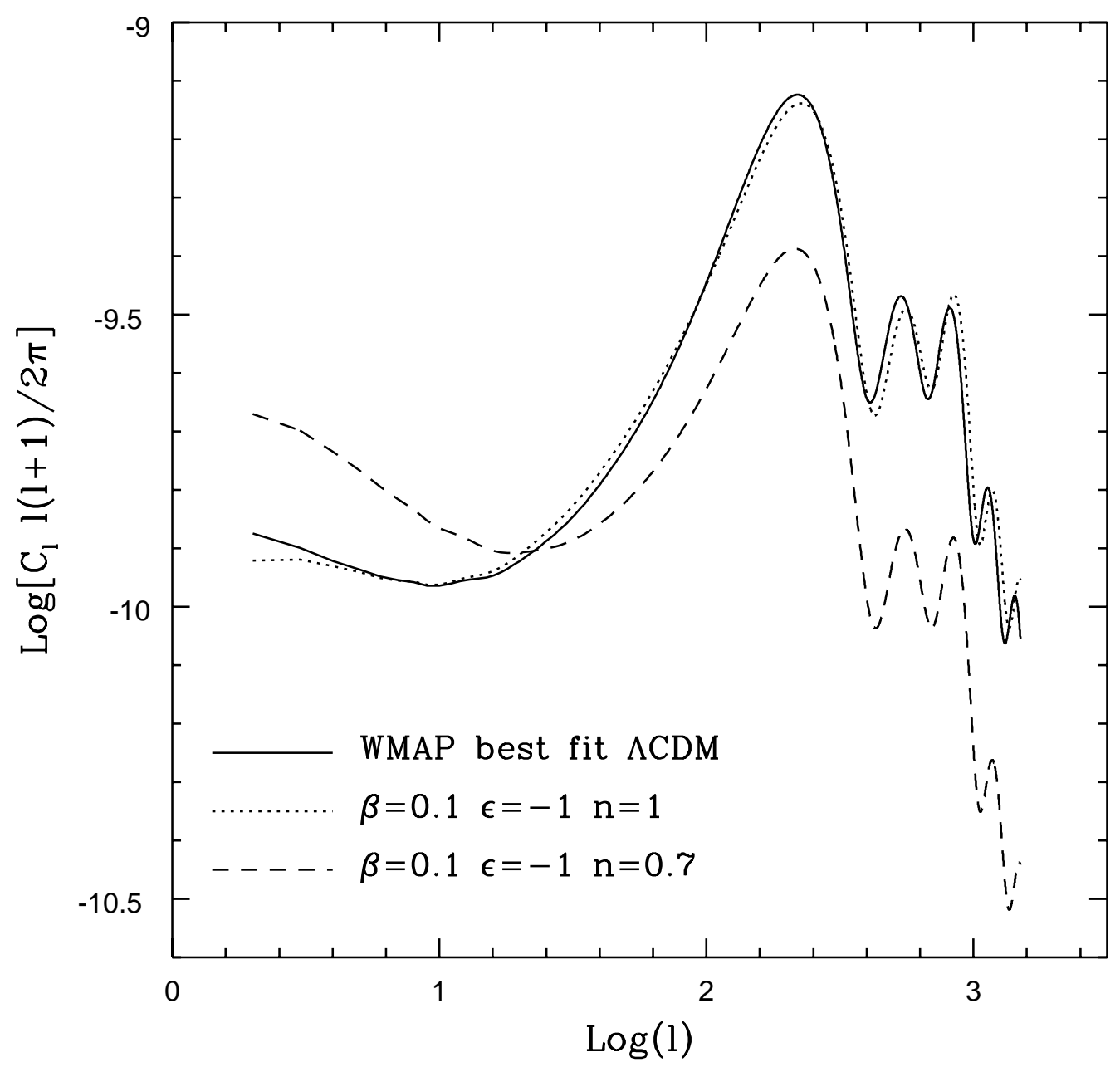

Figure 9. Anisotropy spectrum of the $\Lambda \mathrm{CDM}$ model yielding the best fit to WMAP3 data compared with the spectra for coupled models with $\beta=0.1$ and $\epsilon=1$, for $n=1$

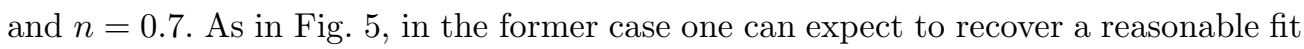
to data by adjusting other model parameters . Taking $n=0.7$, a value just acceptable to fit deep sample data, any fitting to CMB anisotropy data is apparently excluded.

the Sachs \& Wolfe plateau, while reducing the relative height of the $C_{l}$ peaks (see again Fig. 9. These effects could be partially compensated by an adjustment of other model parameters, whose search is out of the scopes of this work. Finding $n$ values below $0.8-0.9$, however, clearly means that we are dealing with unlikely physical frameworks, hardly allowing to fit CMB and deep sample data simultaneously.

In the case $\epsilon \neq 0$, the discrepancy from unity of the spectral index $n$, assumed to be constant, is mostly a measure of the distortion caused by the suppression of Meszaros effect, which overwhelms the effects of the displacement of $k_{h o r, e q}$, already considered in the $\beta=$ const. case. Moreover, such discrepancy is a significant estimate of the distance of the model from uncoupled physics. Our fits, shown in Figures 10 and 11, 


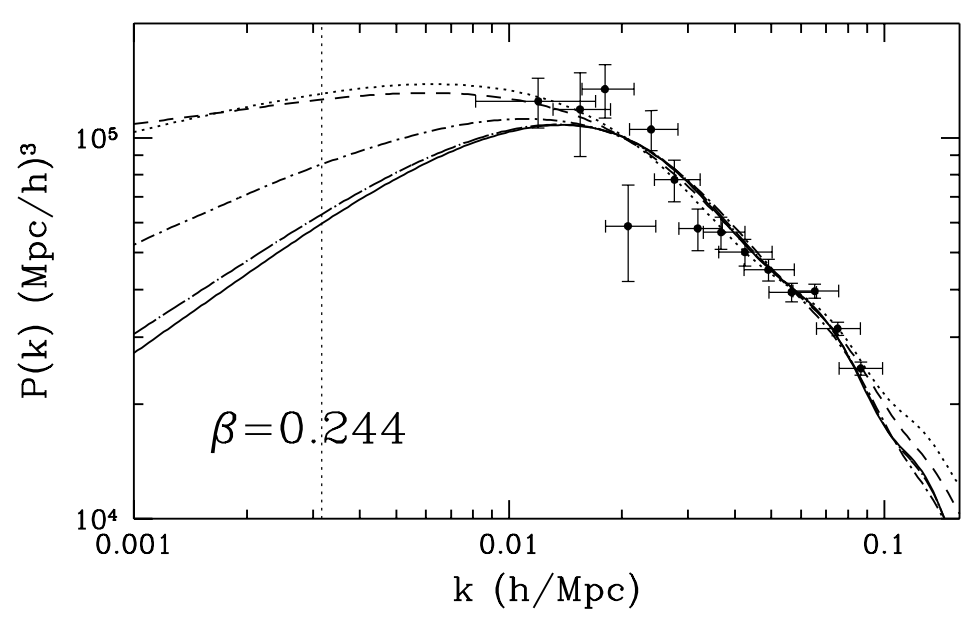

Figure 10. Model comparison with SDSS digital survey data. Different curves refer to different values of $\epsilon$ (as in previous Figure) with the solid line $(\epsilon=1)$ essentially coinciding with an uncoupled model. Constant coupling models $(\epsilon=0)$ are described by dot-dashed curves. Negative $\epsilon$ 's yield a further decrease of $n$. The vertical dotted line is the approximate scale where the Sachs \& Wolfe $C_{l}$ plateau begins. Constant coupling causes a rise of $C_{10}$ by a factor $\sim 1.8$. A further factor $\sim 2$ arises from a coupling $C=1 / \phi$.

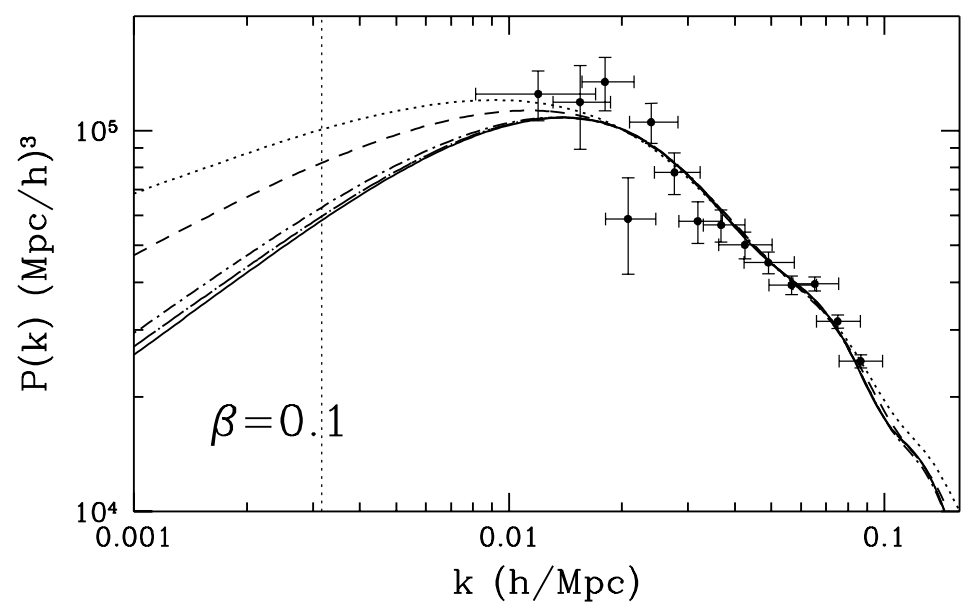

Figure 11. As fig. 10, for a smaller coupling intensity. The effect on the $C_{10}$ scale is much reduced, for constant coupling; however, variable coupling yields a dramatic decrease of $n$ and, for $\epsilon=-1$, the level of $\beta=0.244$ is almost attained.

are complemented by $1-$ and $2-\sigma$ intervals around best-fit $n$ values (Figure 12); they are an indication of which models, by adjusting other parameters, might be susceptible to approach the observational scenario. The behavior of transfered spectra in respect to data, when taking $n$ values at $1-$ and $2-\sigma$ from best fits is also shown in Figure 13 (similar to Fig. 3).

If we take again $n=0.85$ at $1-\sigma$ as a threshold to discard a model, no $\epsilon<0$ coupled model is allowed with $\beta=0.244$, while $\epsilon<-0.16$ are also inhibited with $\beta=0.1$. At 

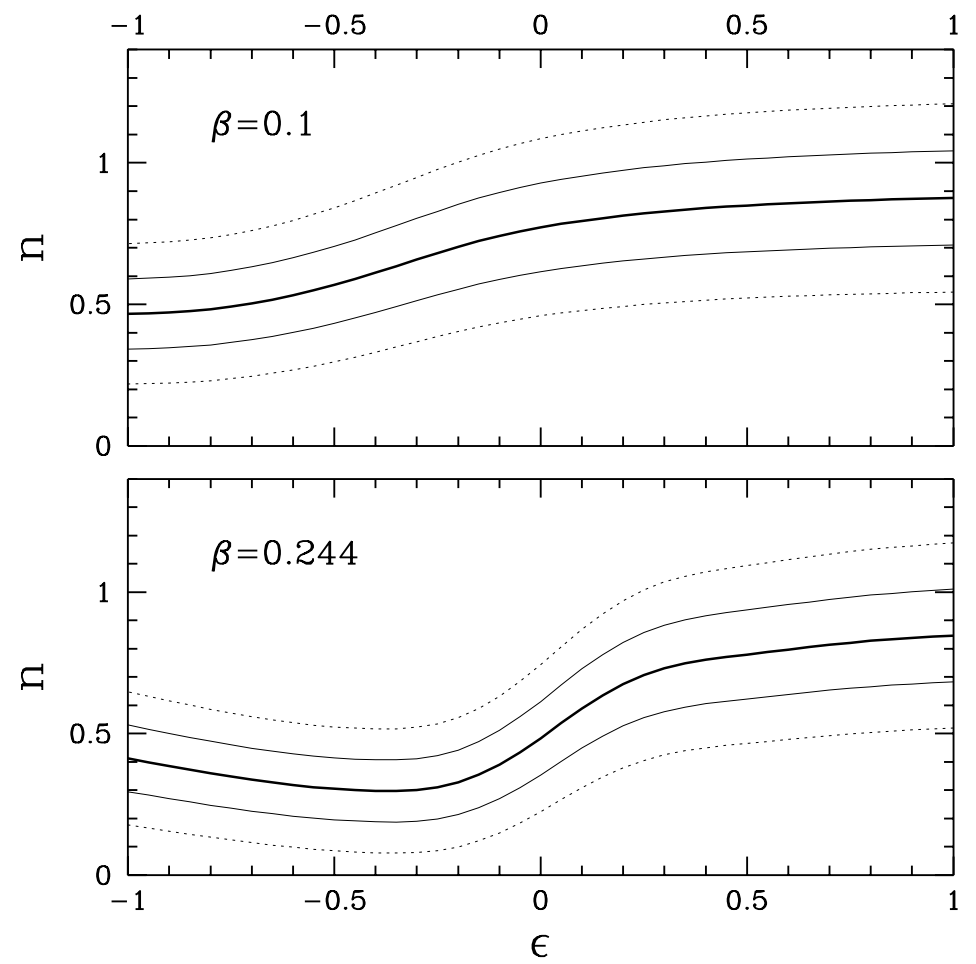

Figure 12. $1-$ and $2-\sigma$ intervals of $n$, when $\epsilon$ varies, for $\beta$ values.

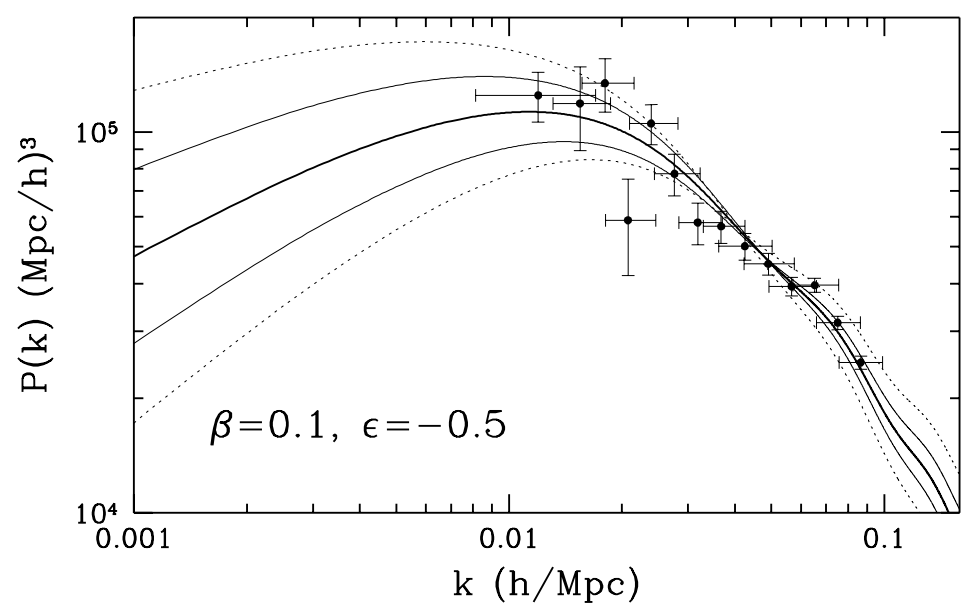

Figure 13. If values of $n$ at $1-$ or $2-\sigma$ 's from best fits are taken, spectra are significantly modified. Here we show the effect in the case with $C=0.4(\pi / 3)^{1 / 2} / \sqrt{m_{p} \phi}$. 
$2-\sigma$ 's the situation is not much improved for $\beta=0.244$, while lower values of $\epsilon$ are admitted for $\beta=0.1$. In particular, a model with $C=1 / \phi$, as the dual-axion model, lays outside of the range indicated.

The analysis was extended here to models with positive $\epsilon$, for which coupling rises while $\phi$ increases. A large deal of these models is apparently allowed.

\section{Conclusions}

The quest for models fitting observational data and avoiding fine tuning and coincidence suggests to test cosmologies where DM is coupled to dynamical DE. It is then important to recognize that quite a few models, with constant or variable DM-DE coupling, are consistent with observational data, although their likelihood might be slightly smaller than uncoupled models.

Constraints on these models were known to arise from a number of linear and nonlinear effects. The main linear anomaly is the existence of a prolongated period, after matter radiation equality, when the $\phi$-field energy affects the expansion rate (the $\phi-$ MD epoch). As a consequence, the comoving distance of the last scattering band can be different from ordinary models. In principle, this can be compensated by varying the present value of the Hubble parameter $H_{o}$. There are however severe limits on $H_{o}$, which turn into limits on the coupling strength, discussed in [9]. The same feature causes also a displacement of the wave number $k_{\text {hor,eq }}$, corresponding to the scale which enters the horizon at matter-radiation equality. Here we discussed also this effect, which was however expected.

Constraints on coupled DE models were also found by studying their non-linear evolution. In [12] it is shown that coupling can affect the halo concentration. Although this effect could be softened by using suitable self-interaction potentials, it yields a limit $\beta<\sim 0.15-0.20$, for the coupling, when the self-interaction potential is Ratra-Peebles [11.

In this paper we outlined a new effect, that DM-DE coupling may cause in a class of models. A prolongated period, between the horizon entry and the equivalence, when DM fluctuation growth stagnates, is essential in shaping transfered spectra $P_{t r}(k)$. Because of this Meszaros's effect, $P_{t r}(k)$ bends at $k>k_{h o r, e q}$. In this paper we outline that this stagnation period can be partially or totally suppressed by the coupling of DM with the $\phi$ field. As a result, transfered spectra exhibit a much softer bending at $k>k_{\text {hor }, e q}$. We also outline that, while this occurs, the dynamics of sonic waves in the baryon-photon fluid is only marginally affected. Accordingly, while transfered spectra suffer major changes, CMB anisotropies are almost invariant.

Using a single primeval spectral index $n$, we can fit both CMB and deep sample data, in the presence of standard Meszaros effect. In the presence of the above softening, the values of $n$ required to fit CMB and deep sample data become badly discrepant.

In this work we discuss which class of coupled DE models are affected by this anomaly. If the coupling is constant or proportional to a positive power of $\phi$, the 
anomaly is absent. On the contrary, models where the coupling is proportional to an inverse power of $\phi$ are at risk.

We gauge the impact of this anomaly by evaluating the value of $n$ required to fit just deep sample data with each model. When $n<\sim 0.85$, it is legitimate to believe that the model likelihood is highly suppressed. The search of such $n$ value should therefore be preliminary to any attempt to reconcile a coupled model to the whole set of CMB, deep sample (and other) data.

Admittedly, apart of conceptual reasons, within the present observational framework there lacks any specific phenomenological push to invoking a DM-DE coupling. Yet, uncoupled models, even apart of their conceptual weakness, cause a number of questionable predictions, e.g. NFW profiles. Interactions within the dark side were often advocated to cure such difficulties. An impact on profiles was actually shown to exist, but new form of coupling need to be inspected.

Furthermore, fresh data on the redshift dependence of $\rho_{c}, \rho_{b}$ and $\rho_{D E}$, at $z \sim 1-5$, might soon be available, if experiments like DUNE [20] will become operational. The discovery of an anomalous scaling of $\rho_{c}$, for instance, would set a strong prior, completely biasing likelihood distributions, just as priors on the value of $h$ (Hubble parameter) suppress the likelihood of SCDM models with $h \sim 0.4$, which would otherwise allow a reasonable fit of large sets of data [21]. In particular, models with rising coupling $(\epsilon>0)$ could become an important option. Such coupling behavior could directly arise from suitable microphysics or be a phenomenological description of a complex underlying physics.

\section{Acknowledgments}

Luca Amendola and Loris Colombo are gratefully thanked for their comments on this work. 


\section{References}

[1] Wetterich C. 1988, Nucl.Phys.B 302, 668; Ratra B. \& Peebles P.J.E., 1988, Phys.Rev.D 37, 3406

[2] Peebles P.J.E. \& Ratra B., 2003, Rev.Mod.Phys. 75, 559

[3] Kolb E.W., Matarrese S. \& Riotto A., 2006, New J.Phys. 8, 322

[4] Ellis J., Kalara S., Olive K.A. \& Wetterich C., 1989, Phys. Lett. B228, 264; Wetterich C., 1995, A\&A 301, 321 Amendola L., 2000, Phys.Rev. D62, 043511 Gasperini M., Piazza F.\& Veneziano G., 2002, Phys.Rev. D65, 023508

[5] Casas J.A., Garca-Bellido J \& Quiros M., 1992, Class.Quant.Grav. 9, 1371; Anderson G.W . \& Carroll S.M., Procs. of "COSMO-97, First International Workshop on Particle Physics and the Early Universe", Ambleside, England, September 15-19, 1997, astro-ph/9711288; Bartolo N. \& Pietroni M., 2000, Phys.Rev. D61, 023518; Pietroni M., 2003, Phys.Rev D67, 103523; Chimento L.P., Jakubi A.S., Pavon D. \& Zimdahl W.,2003, Phys.Rev D67, 083513; Rhodes C.S., van de Bruck C, Brax P., \& Davis A.C., 2003, Phys.Rev. D68, 083511; Farrar G.R. \& Peebles P.J.E., 2004, ApJ 604, 1 Gromov A., Baryshev Y. \& Teerikorpi P., 2004, A\&A, 415, 813

[6] Mainini R. \& Bonometto S.A., 2004, Phys.Rev.Lett. 93, 121301

[7] Peccei R.D. \& Quinn H.R. 1977, Phys.Rev.Lett. 38, 1440; Weinberg S. 1978, Phys.Rev.Lett. 40, 223; Wilczek F. 1978, Phys.Rev.Lett. 40, 279; Kim J.E. 1979, Phys.Rev.Lett. 43, 103

[8] Preskill J. et al 1983, Phys.Lett B120, 225; Abbott L. \& Sikivie P. 1983 Phys.Lett B120, 133; Dine M. \& Fischler W. 1983 Phys.Lett B120, 137; Turner M.S. 1986 Phys.Rev.D 33, 889

[9] Mainini R., Colombo L. \& Bonometto S.A., 2005, ApJ 632, 691

[10] Amendola L. \& Quercellini C., 2003, Phys. Rev. D68, 023514

[11] Ratra B. \& Peebles P.J., 1988, Phys.Rev. D37, 3406

[12] Maccio' A. V., Quercellini C., Mainini R., Amendola L., Bonometto S. A., 2004, Phys. Rev. D69, 123516

[13] Spergel D.N. et al. 2006, ApJ (in press), astro-ph/0603449

[14] Brax P. \& Martin J., 1999, Phys.Lett., B468, 40; Brax P. \& Martin J., 2001, Phys.Rev. D61, 10350; Brax P., Martin J., Riazuelo A., 2000, Phys.Rev. D62, 103505

[15] Colombo L.P.L. \& Gervasi M., 2006, JCAP 0610, 001

[16] Coles M. \& Lucchin F., 1995, Cosmology, John Wiley \& Sons, Chichester

[17] Lee S., Liu G. \& Ng K., 2006, Phys.Rev. D73, 083516; Guo Z., Ohta N. \& Tsujikawa S., 2007, astro-ph/0702015

[18] Tegmark M. et al., 2006, Phys.Rev. D74, 123507

[19] Amendola L., 2004, Phys.Rev.D69, 103524

[20] Réfrégier A. et al., Procs. of SPIE symposium "Astronomical Telescopes and Instrumentation", Orlando, may 2006, astro-ph/0610062

[21] Blanchard A., Douspis M., Sarkar S., Rowan-Robinson M., 2003, A\&A 412, 35 